\title{
Investigation on the Association of Soil Microbial Populations with Ecological and Environmental Factors in the Pearl River Estuary
}

\author{
Yanchun Qiao1*, Donglin Xu1 ${ }^{*}$, Hongjuan Yuan2 ${ }^{2}$, Bei Wu1', Bishao Chen1, Yaowen Tan1, \\ Jianrong Lin ${ }^{1}$, Dongliang Guo ${ }^{3 \#}$ \\ ${ }^{1}$ Guangzhou Academy of Agricultural Sciences, Guangzhou, China \\ ${ }^{2}$ Department of Life Science, Yuncheng University, Yuncheng, China \\ ${ }^{3}$ Institute of Fruit Tree Research, Guangdong Academy of Agricultural Sciences, Guangzhou, China \\ Email: ${ }^{5}$ 16986554@qq.com
}

How to cite this paper: Qiao, Y.C., Xu, D.L., Yuan, H.J., Wu, B., Chen, B.S., Tan, Y.W., Lin, J.R. and Guo, D.L. (2018) Investigation on the Association of Soil Microbial Populations with Ecological and Environmental Factors in the Pearl River Estuary. Journal of Geoscience and Environment Protection, 6, 8-14.

https://doi.org/10.4236/gep.2018.63002

Received: January 22, 2018

Accepted: March 10, 2018

Published: March 13, 2018

\begin{abstract}
An investigation on the soil microbe populations from an agricultural inning area at the Pearl River estuary in Guangdong province, China was conducted via high through-put sequencing. The results revealed abundant diversity in the soil bacterial and fungal populations. In total, 197103 sequence tags were obtained from soil samples, most of which represented bacterial genera Actinomycetes, Bacillus and Marinobacter, while a majority of 118378 tags obtained were derived from fungal genera Clostridium, Devosia, Bradyrhizobium, Bdellovibrio, Phenylobacterium, Penicillium, and Emericella. Furthermore, nine physiological indexes $(\mathrm{pH}$, available phosphorous, basic-group nitrogen, available potassium, catalase, sucrose, urease, phosphatase, and organic matters) were measured in three soil samples, and the association between these physiological indexes and microbe population composition was examined. The results revealed obvious inter-sample differences associated with ten dominating microbial groups: genera Clostridium, Devosia, Bradyrhizobium, Bdellovibrio, Phenylobacterium, and Penicillium were mainly impacted by $\mathrm{pH}$ (with a positive correlation), genera Sphingomonas and Acinetobacter mainly by available phosphorous (positive correlation), and genera Gemmatimonas and Pseudomonas by both $\mathrm{pH}$ and available phosphorous (negative correlation). Our study suggested that regulation of microbial species/populations might help improve soil environment to facilitate the growth of crops' above-ground parts, and this provides practical information for inning agriculture.
\end{abstract}




\section{Keywords}

High Through-Put Analysis, Pearl River Estuary, Soil Microbial Population, Environmental Factors, Association

The Pearl River delta, an area of advanced social economy development in Guangdong province, China, enjoys prominent agricultural resource advantages: fertile land, sufficient irrigation, and year-round climate conditions suitable for crop growing. The delta has therefore been one of the most important centers of rice, sugarcane, and fruit production, sericulture, and pond aquaculture. In recent years, the growing urbanization has drawn the government's attention to protection and exploitation of the agricultural inning and intertidal zones. In December 2012, the provincial government issued "The Plan for Protection and Exploitation of Intertidal Zones at the Pearl River Estuary", which mentioned the necessity of vegetable cultivation in the future-exploited intertidal zones to the public supply of vegetables for the city. To meet up the new need for vegetable breeding research, our institute, Guangzhou Academy of Agricultural Sciences (GZAAS), will move its vegetable breeding and production canter from its Huadu base to the Nansha base this year. The Huadu base is located in the northern part (Huadu district) of Guangzhou city and characteristic of sandy loam, whereas the Nansha base, in the southern part (Nansha district) of the city, is located at the estuary of the Pearl River characteristic of alluvium (clay loam) under seasonal influence of saline tide, which forms a special microbiological environment. To investigate the soil microbial populations from these two vegetable bases and their association with the environmental factors will provide important insight into the adaptation and sustainable development of vegetable industry in Guangzhou city.

\section{Materials and Methods}

\subsection{Sample Collection}

The 20-cm depth soil samples were collected from the GZAAS Huadu and Nansha bases, respectively, according to a nine-point sampling method, well-mixed and air dried in our laboratory. The dried soil then went through a 0.15 micrometer, 300 -mesh sieve and was stored at $-20^{\circ} \mathrm{C}$, for the uses of soil microbe investigation and analysis of physical and chemical soil properties.

\subsection{High Through-Put Sequencing and Analysis of Soil Microbial Population}

The DNA of soil samples were extracted using a soil DNA extraction kit after they went through a sieve. The obtained DNA was subject to quality verification, PCR amplification (16S rDNA V3-V4 region for bacteria and ITS2 region for fungi) and then high through-put sequencing of the PCR products by Guang- 
zhou Gene Denovo Bio-Tech Ltd. Co. (Guangzhou, China). The obtained data were assembled into sequence tags and subject to BLAST in GenBank for classification of the microbes, followed by OTU, diversity and inter-sample comparative analyses.

\subsection{Analysis of Association between Dominating Microbial Populations and Soil Physiological Indexes}

Nine environmental factors $-\mathrm{pH}$, available phosphorous, basic-group nitrogen, available potassium, hydrogen peroxide, sucrose, urease, phosphatase, and organic matters-were measured in the Huadu and Nansha soil samples using standard protocols, with an original uncultivated soil sample (maintained in our laboratory) as the control. A canonical correspondence analysis (CCA) was conducted to reveal the association between the dominating microbial populations (on the genus level) in the soil samples and these factors.

\section{Results}

\subsection{High Through-Put Sequencing of Soil Microbes from the Two Bases}

32.9 and 33.0 Mbp nucleotides from fungal ITS2 regions were obtained from the Huadu and Nansha samples respectively, and then the clean data were assembled into 117,012 and 118,378 sequence tags, respectively. The Huadu sequences ranged 201 - $349 \mathrm{bp}$, and the Nansha ones $201-349$ bp in length. For the bacterial 16S rDNA regions, 61.6 and 68.2 Mbp nucleotides containing 204,507 and 194,103 unique tags, respectively, were obtained from the Huadu and Nansha samples.

A GenBank BLAST analysis of the assembled sequences indicated that in the Huadu but not Nansha sample, abundant sequences of Fusarium (e.g. F. oxysporum), known as a plant pathogenic fungal genus, were identified, whereas fungi from genera Clostridium, Devosia, Bradyrhizobium, Bdellovibrio, Phenylobacterium, Penicillium, and Emericella dominated in the Nansha soil sample, and the Emericella sp., Penicillium menonorum and P. pimiteouiense were found in the Nansha but not in the Huadu sample (Figure 1). A terpenoid, Emericella-sp-d01, has been found as a seaborne fungus-derived secondary metabolite with antibiotic activity. $P$. menonorum may promote plant growth and nutrient management, while $P$. pimiteouiense play the roles as calmodulin and maintenance factor for plants. It is suggested that these three particular microorganisms are associated with the special soil environment in the Nansha base under constant influence of saline tide, and that they may be conducive to the growth of crops' above-ground parts.

Both the Huadu and Nansha samples contained diverse bacterial groups. The dominating genera in the two samples were Sphingomonas and Acinetobacter respectively, and genera Actinomycetes, Bacillus and Marinobacter were present with different abundances in both samples (Figure 2). The Huadu sample 


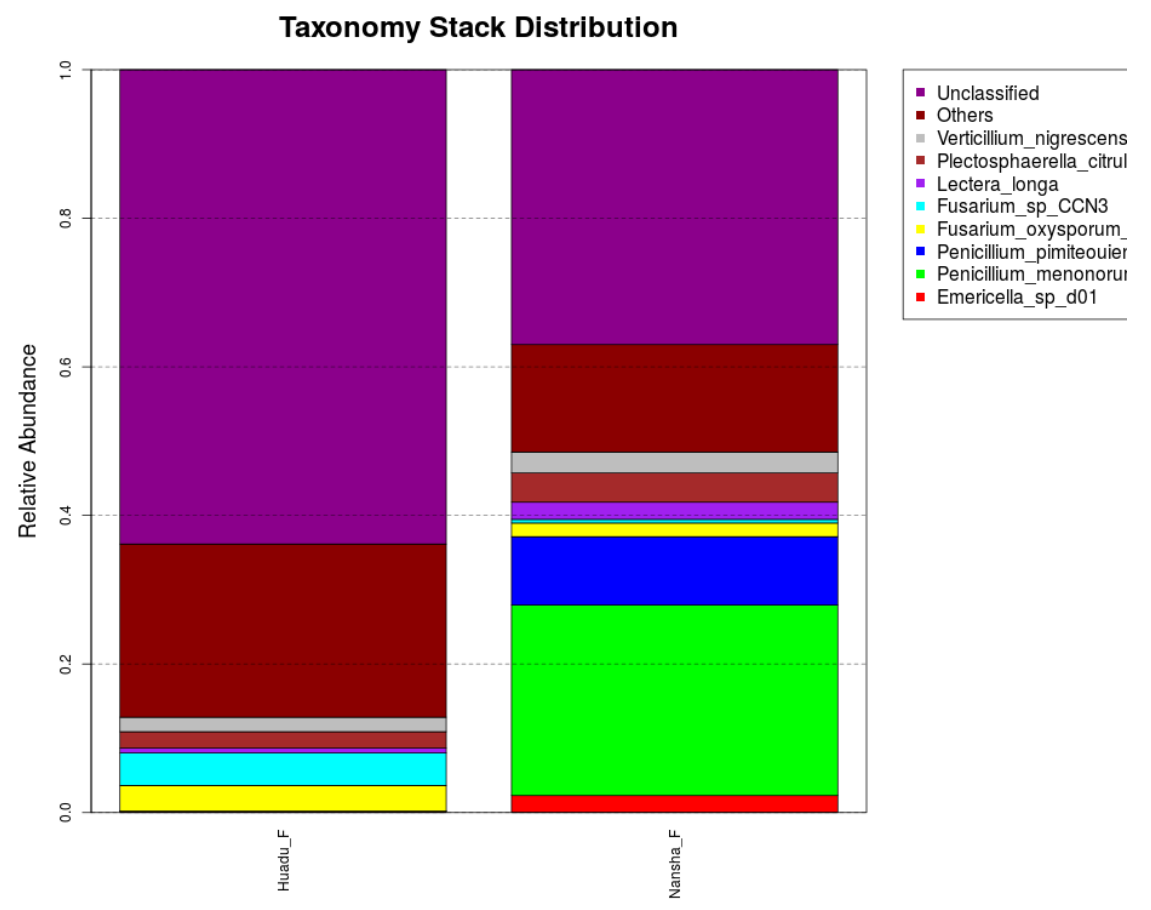

Figure 1. Distribution of fungal species detected in soil samples from the Huadu and Nansha bases.

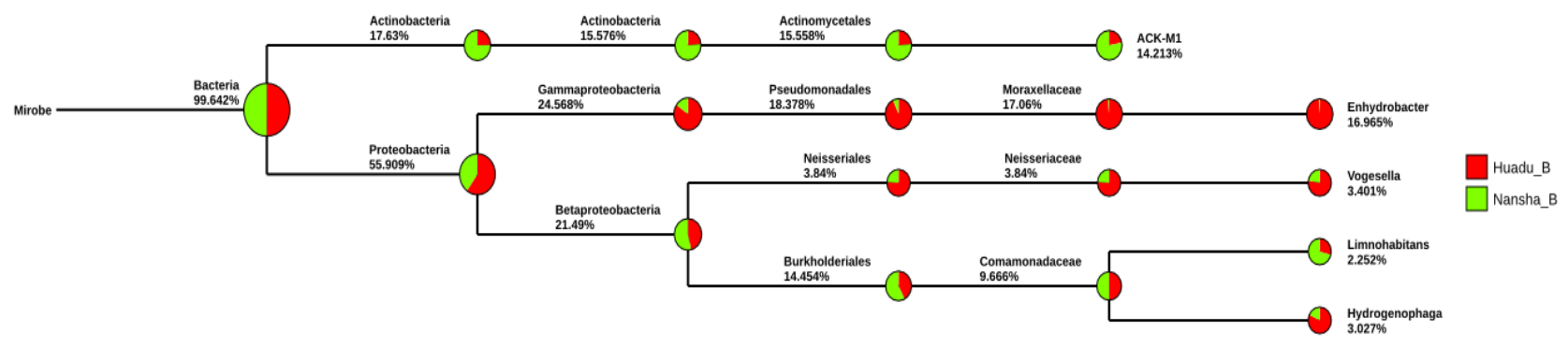

Figure 2. Distribution of bacterial groups detected in soil samples from the Huadu and Nansha bases.

generated 2260 Bacillus tags, significantly more than the Nansha sample (18 tags). In contrary, the Nansha sample contained much abundant Actinomycetes (52828 tags) and Marinobacter (21978 tags)bacteria than the Huadu sample (17976 and 8628 tags respectively). It has been reported that higher Bacillus titeris harmful while Actinomycetes is beneficial, to plant growth [1] [2], suggesting that the soil environment in the Nansha base is more suitable for vegetable plantation than the Huadu base.

\subsection{Test of Physiological Indexes for the Soil Samples}

Compared to the control, both the Huadu and Nansha soil samples had higher $\mathrm{pH}$ and contents of eight types of indexing substances: available phosphorous, basic-group nitrogen, available potassium, catalase, sucrose, urease, phosphatase, and organic matters (Table 1 ). Both the samples were weekly acid ( $\mathrm{pH}$ 6.16 and 6.22). The Nansha sample contained much lower amounts of available 
Table 1. Nine physiological indexes tested for the soil samples from the Huadu and Nansha bases.

\begin{tabular}{|c|c|c|c|c|c|c|c|c|c|}
\hline Sample & $\begin{array}{c}\mathrm{pH} \\
(\text { water:soil }=5: 1 \text { ) }\end{array}$ & $\begin{array}{c}\text { Available } \\
\text { phosphorous } \\
(\mathrm{mg} / \mathrm{Kg})\end{array}$ & $\begin{array}{c}\text { Basic-group } \\
\text { nitrogen }(\mathrm{mg} / \mathrm{Kg})\end{array}$ & $\begin{array}{c}\text { Available } \\
\text { potassium }(\mathrm{mg} / \mathrm{Kg})\end{array}$ & $\begin{array}{c}\text { Catalase } \\
(\mathrm{ml} / \mathrm{g})\end{array}$ & $\begin{array}{l}\text { Sucrose } \\
(\mathrm{mg} / \mathrm{g})\end{array}$ & $\begin{array}{l}\text { Urease } \\
(\mathrm{mg} / \mathrm{g})\end{array}$ & $\begin{array}{c}\text { Phosphatase } \\
\text { (mg/g) }\end{array}$ & $\begin{array}{l}\text { Organic matters } \\
(\mathrm{g} / 100 \mathrm{~g})\end{array}$ \\
\hline Huadu & 6.16 & 345.20 & 384.76 & 417.72 & 1.25 & 10.98 & 9.77 & 109.18 & 23.00 \\
\hline Nansha & 6.22 & 225.02 & 292.30 & 469.06 & 1.72 & 32.54 & 24.58 & 235.55 & 25.30 \\
\hline CK & 4.72 & 153.03 & 168.21 & 201.47 & 0.84 & 8.21 & 7.63 & 86.25 & 10.89 \\
\hline
\end{tabular}

phosphorous and basic-group nitrogen but higher available potassium, catalase, sucrose, urease, phosphatase, and organic matters (Table 1). The result suggest that the saline and alkaline soil in the Nansha base adjacent to the sea can be improved and developed into high-quality plough land via appropriate cultivation measures and use of fertilizers.

\subsection{Analysis of the Association of Soil Microbial Populations with Ecological Indexes}

Our results suggested that the ten microbial genera dominating in the Huadu sample, the Nansha sample, and the control (known with Gemmatimonas, Pseudomonas, and Paenibacillus dominating) respectively were influenced by the nine environment factors. The canonical correspondence analysis (CCA) indicated that the microbes from genera Clostridium, Devosia, Bradyrhizobium, Bdellovibrio, Phenylobacterium, and Paenibacillus were mainly impacted by $\mathrm{pH}$ in a positive correlation; that from genera Sphingomonas and Acinetobacter mainly by available phosphorous content in a positive correlation; and that from genera Gemmatimonas and Pseudomonas by both $\mathrm{pH}$ and available phosphorous content in a negative correlation (Figure 3 ).

\section{Discussion}

Our study explored the dominating microbial populations in soil samples from two vegetable breeding and cultivation bases located in northern and southern parts of Guangzhou city, Guangdong province, the latter (Nansha base) being on the Pearl River estuary with its own special environment. Our results indicated different soil microbial populations in the two locations and revealed their association with the very distinct physiological indexes of the soil samples from these two bases. This provides useful information for inning agriculture, for it is implied that soil environment may be improved by regulation of microbial populations living within to facilitate the above-ground growth of crops.

In this study, Sphingomonas was found dominating in the soil from the Huadu base, while genera Clostridium, Devosia, Bradyrhizobium, Bdellovibrio and Phenylobacterium dominated in the Nansha soil environment. It has been reported that Sphingomonas is a group of bacteria widely distributed in soil that can degrade aromatic pollutants [3]. The Huadu base and Baiyun International Airport are both located in the Huadu district, Guangzhou. Deposition of pollutants 


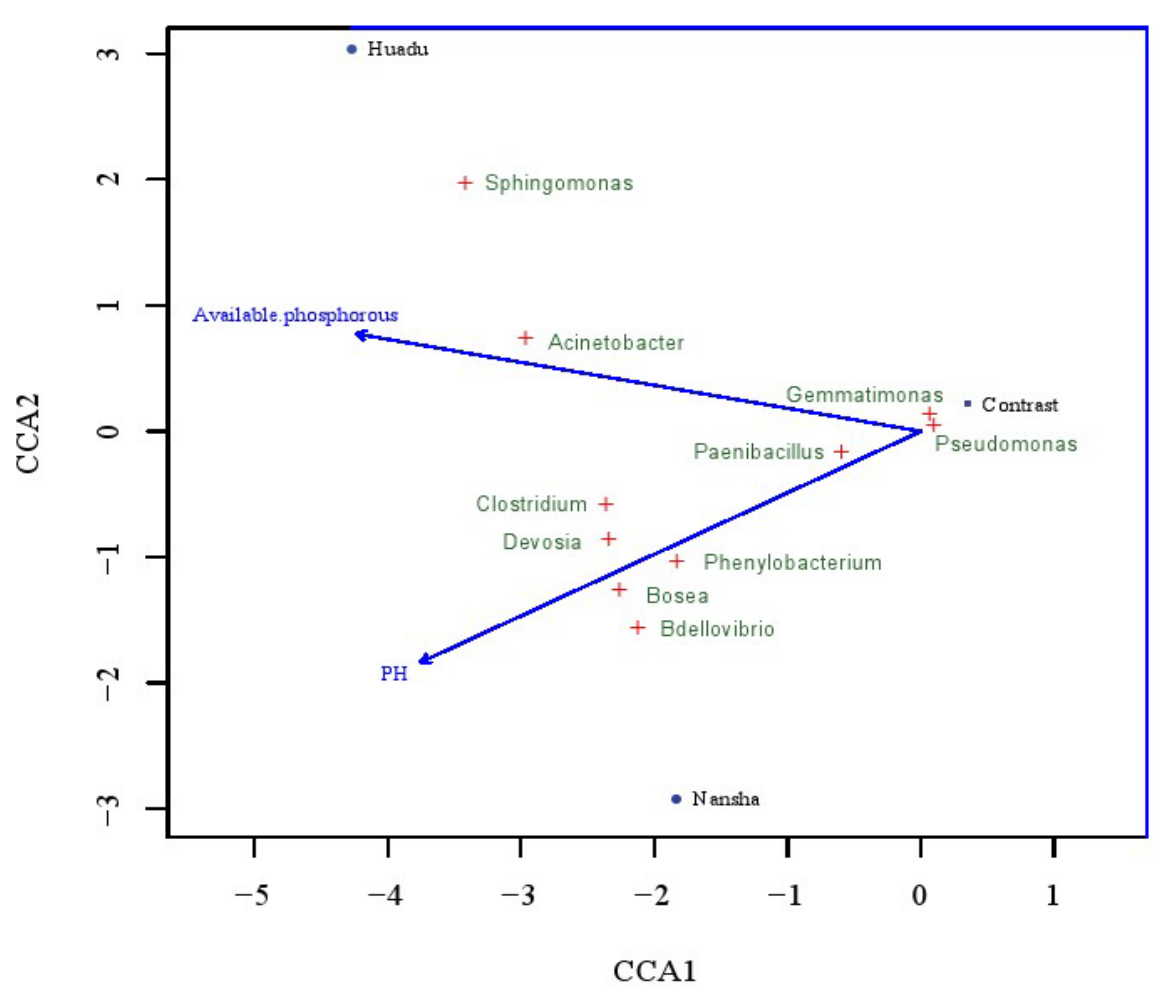

Figure 3. Canonical correspondence analysis of the association between nine physiological indexes and microbe populations from the Huadu and Nansha samples.

coming from aircraft fuel exhausts might have caused the thriving of Sphingomonas in the Huadu soil environment. For the five dominating microbial populations in Nansha, except genus Clostridium, a type of human pathogenic microbes [4], the other four are beneficial factors to the environment: Devosia may degrade a vomit-causing toxin (vomitoxin or DON) into nontoxic substances [5]; Bradyrhizobium are a group of nitrogen-fixing bacteria beneficial for nutrition utilization of plants [6]; Bdellovibrio are a class of bacterium-parasitic bacteria that may cause cell lysis of various other bacteria and are believed to be a natural purifying biological factor [7]; and bacteria of genus Phenylobacterium may degrade herbicides like acetochlor [8] These suggest that the Nansha base will be environmentally superior to the Huadu base for vegetable breeding and cultivation and agricultural production.

\section{Acknowledgements}

We would like to thank the Project of Science \& Technology Plan of Guangdong Province (2013A061401019) and Supportive Fund for Agriculture of Guangzhou City (2014-2018) for financial support.

\section{References}

[1] Li, F.Y., Wang, Y. and Hu, X.H. (2013) Screening of the Optimai Applying Concentration of Actinomycetes in Continus Tomato Cropping Soil. Acta Agriculturae Boreali, 22, 132-136. 
[2] Wei, H.Y., Cai, L.M., Zhao, Y.Y. and Tan, T.Y. (2009) Effect of Bacillus subtilis on Microbial Respiration and Urease Activity in Phaeozem. Agrochemicals, 48, 111-113.

[3] Gou, M., Qu, Y.Y., Yang, H., Zhou, J.T., Li, A., Guan, X.Y. and Ai, F.F. (2008) Sphingomonas sp: A Novel Microbial Resource for Biodegradation of Aromatic Compounds. Chin J Appl Environ Biol, 14, 276-282.

[4] Wu, W., Wu, Y. and Qu, J.H. (2011) The Effects of Clostridium Butyricum in Superoxide Dismutase (SOD) and Catalase (CAT) Activity Response to Nitrite Stress in Macrobrachium nipponense. Chinese Agricultural Science Bulletin, 27, 97-102.

[5] Pan, Y.M. (2013) Enzymatic Degradation of 3-Acetyldeoxybivalenol by Devosia sp. dds- 1 and Constructing of the Genomic Fosmid Library. The Master's Degree Thesis of Nanjing Agricultural University.

[6] Cheng, F.X., Cao, G.Q., Wang, X.R., Zhao, J. and Yan, L.H. (2008) Discovery and Application of Efficient Strains of Soybean Rhizobia in Acidic Low-Phosphorus Soil of South China. The Chinese Science Bulletin, 53, 2903-2910.

[7] Zhang, L., Chen, J.Y., Wang, H.W. and Duan, Z. (2004) Effect of Planting Apple Seedlings on the Curtain of Solitary Fish Water Hydration Fade Targets. Henan Fisheries, 3, 34-35.

[8] Zhou, J.J., Zou, F. Yang, H. Xu, J. and Sun, J.G. (2013) Screen for Acetochlor Degrading Bacteria and Identification. The Chinese Soil and Fertilizer, No. 6, 93-96. 